\title{
دراسة تحليلية للميزان التجاري في الجزائر
}

\author{
عحم علي سكران محزم السهلي \\ باحث دكتوراه بمعهد البحوث والدراسات الأفريقية - جامعة أسوان
}

محمد عبد الحفيظ محمد

أستاذ الإقتصاد الزراعى - كلية الزراعة - جامعة سوهاج

حسن موسي رضوان

مدرس الاقتصاد الزراعي بالمعهد العالي للتعاون والإرشاد الزراعي بأسيوط رون 


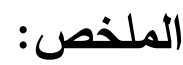

لقد تتاولنا في هذا البحث تحليلة الميزان التجاري، من خلال أسباب قيام التبادل

الدولي، و الغاية من تحرير قطاع التجارة الخارجية و أفضل السبل لذلك. وقد حقق

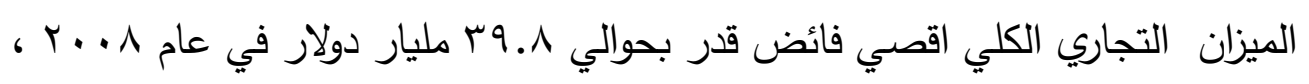
وعجز قدر حوالي ا. . ب ملياردولار في عام 7 ـ ـr ، وبلغ متوسط الفائض خلال فترة الدراسة حوالي ؟^.^ مليار دولار .

ويعبر مؤشر معدل التغطية للتجارة للخارجية بصفة أساسية عن كفاءة التجارة

الخارجية الكلية، وقدرتها على تغطية نفقات الواردات من حصيلة الصادرات ، كما يشير ايضا الى الوضع النسبي للجزئر في عملية التبادل الدولي أي مدى تحكمها في وارداتها، أن معدل تغطية الصادرات الكلية للواردات الكلية تراوح بين حد أدني بلغ

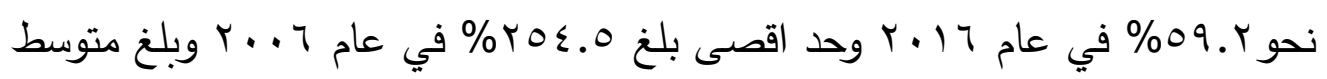
معدل تغطية الصادرات للواردات خلال فترة الدراسة نحو ؟ ., بس \% ـ مما يشير الى وجود فائض كبيرة بين قيمة الصادرات الكلية وقيمة الواردات الكلية تكون في صالح الموازنة التجارية بالدولة، وأنها تمثل اعتماداً على الخارج. الكلمات الإفتتاحية: الميزان التجارى - الصادرات - الواردات - الجزائر

\section{Abstract}

Analytical Study of the Trade Balance in Algeria 
The overall trade balance achieved a maximum surplus of about $\$ 39.8$ billion in 2008 , and a deficit of about $\$ 20.1$ billion in 2016 , and the average surplus during the study period was about $\$$ 8.84 billion.

The index of the rate of coverage of foreign trade mainly reflects the efficiency of the total foreign trade and its ability to cover imports expenditures from the export proceeds, also indicates the relative position of Algeria in the international exchange process, i.e. the extent of its control over its imports, that the rate of total exports coverage of total imports ranged between a limit The lowest was about $59.2 \%$ in 2016 , and a maximum of $254.5 \%$ in 2006. The average rate of export coverage of imports during the study period was about $132.04 \%$. Which indicates that there is a large surplus between the value of total exports and the value of total imports, which is in favor of the state's trade budget, and that it represents dependence on the outside. 
تلعب التجارة الخارجية دورا هاما في معظم الاقتصاديات الدولية حيث توفر للاقتصاد ما تحتاج إليه من سلع وخدمات غير متوفرة محليا، من خلال نثاط الاستيراد وفي نفس الوقت تمكنه من التخلص ما لايه من فائض من السلع والخدمات المختلفة من خلال نشاط التصدير تعتبر العمليات الاقتصادية سوآءا الوطنية أو الدولية محركا أساسيا لنشاط التجارة الخارجية وترجع هذه الصفقة التي تتميز بها التجارة الخارجية إلى الإنسان ورغبته، وهذا ليلبي ما يحتاج إليه في حياته اليومية المتزايدة وباستمرار ، فالتجارة ماهي إلا مجموعة من العلاقات التي تربط بين الأعوان الاقتصاديين الدقمين والأعوان الاقتصاديين غير الsقمين وذلك في مختلف دول العالم. وبما أن العالم يسعي اليوم بكافة الطرق الي التوسع في التجارة الخارجية للأهية البالغة التي تكتسبها هذه الأخيرة إضافة الي أن المناخ الإقتصادي الدولي في تطور مستمر نحو الاتحاد والتكامل الاقتصادي مما استوجب علي الدول النامية ومن بينها الجزائر مواكبة هذا التطور الحاصل. الجزائر كباقي دول العالم التي سعت لمواكبة هذا التطور وبعد أن ورثت عقب استقلالها نظاما سيطر علية اقتصاد فرنسا في مجال المبادلات التجارية ولكون قطاع التجارة الخارجية المتنفس الوحيد لما يوفره من مدخلات تسمح بتغطية متطلبات الدولة إعتمادا علي صادراتها من المواد الأولية ( البترول والغاز)، رأت الدولة أن تسير هذا القطاع بانتهاج سياسات مختلفة تتلاءم مع المرحلة الإقتصادية التي تمر بها البلاد 
فعددت خلال مسيرتها التتموية الي فرض الرقابة علي التجارة الخارجية في المرحلة أولي ثم احتكارها في المرحلة ثانية، ولكن بعد أزمة الدولار وأزمة النفط في عام 1919 وانخفاض اسعار الدحروقات التي انعكست بنقص إيرادات الدولة من التجارة الخارجية، حيث تعتبر الدحروقات العنصر الأساسي في الايرادات الدولة من التجارة الخارجية. إثكالية البحث:

رغم الأهمية التي يكتسبها الميزان التجاري كقوة دافعة للتمية الاقتصادية الا أن

هنآك بعض المتغيرات والتحولات الاقتصادية التي لها أثر علي وضعيته وبالتالي يجب أخذها في الحسبان، ماهي أهم التحليلات التي يمكن أن نقدمها للتغيرات التي مست

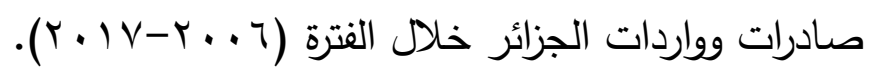

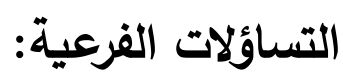

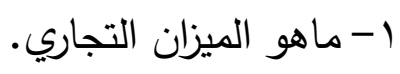

r- ماهو واقع تطبيق استراتيجات كل من الاستيراد والتصدير في الجزائر.

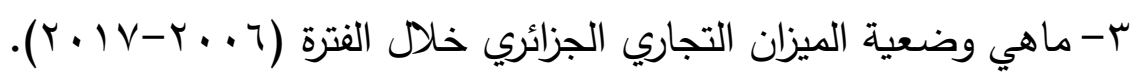

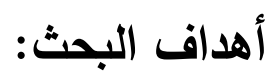

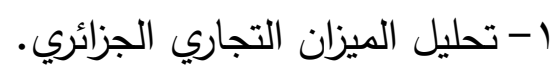
ץ-معرفة مدي تأثر وضعية الميزان التجاري الجزائري بالمتغيرات والتحولات الاقتصادية. 
r-معرفة الدور الذي تلعبه كل من الصادرات والواردات في تحديد وضعية الميزان

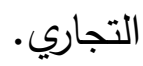

\section{منهج ووسائل جمع البيانات للدراسة:}

اعتمدت الدراسة علي الدنهج الوصفي، واستخدامها في إبراز أهم الاحصائيات

التي تخص الميزان التجاري الجزائري من خلال تحليل التركيبة السلعية لكل من

$$
\text { الصادرات والواردات. }
$$

حدود الاراسة:

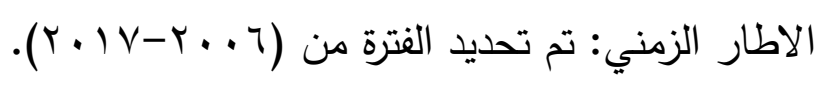

الاطار المكاني: تستهدف الدراسة الجزائر.

الاطار الموضوعي: الدراسة الحالية تقتصر علي دراسة وتحليلة الميزان

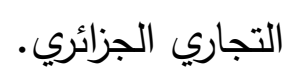

\section{نتائج الاراسة:}

يقصد بالميزان التجاري رصيد العمليات، اي المشتريات والمبيعات من السلع والخذمات، وهذا هو المعني الواسع للميزان التجاري. كما يعرف الميزان التجاري هو الفرق بين قيم الصادرات والواردات من السلع والخدمات خلال فترة زمنية معينة.وهكذا تقيم العلاقة بين صادرات وواردات البلد ويعبر عنها بالمعادلة التالية: 


$$
\text { الميزان التجاري= إجمالي صادرات - إجمالي الواردات }
$$

\section{أقسام الميزان التجاري:}

أ- الميزان التجاري السلعي: يطلق علية أيضا ميزان التجارة المنظور، ويضم كافة السلع والخدمات التي تتخذ بشكل ماديا ملموسا( الصادرات والواردات من السلع

$$
\text { المادية التي تتم عبر الحدود الجمركية. }
$$

ب-الميزان التجاري الخدمي: ويطلق عليه ايضا الميزان التجاري غير النظورة، وتضم كافة الخدمات المتبادلة بين الدول ( النقل والسياحة و التأمين و دخول العمل و

$$
\text { عوائد رأس المال). }
$$

\section{المبحث الأول: تحليل تطور الصادرات والواردات في الجزائر:}

سنحاول من خلال هذا المبحث تحليل تطور الصادرات والواردات في الجزائر من ناحية ، وتطور التركيبة السلعية للصادرات، وتطور التركيبة السلعية للواردات في

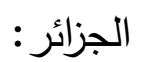

المطلب الأول: تحليل تطور الميزان التجاري في الجزائر:

تشير البيانات الواردة في جدول رقم ( ) والشكل رقم (1) إلي أن إجمالي قيمة التجارة الخارجية الكلية للجزائر خلال فترة الدراسة تراوحت بين حد ادني بلغ حوالي

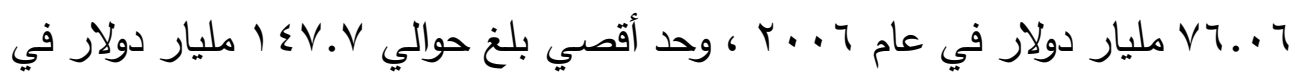




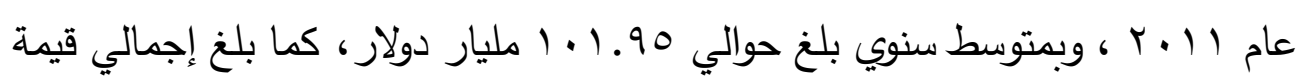
صادارتها الكلية في نفس الفترة ما بين حد ادني قدر بحوالي ب.؟Y مليار دولار في عام

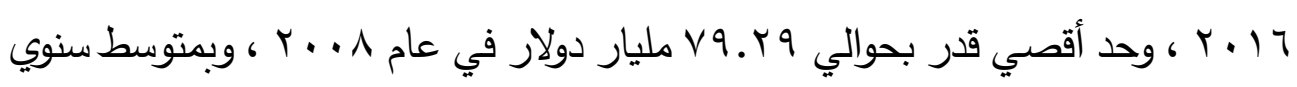
بلغ حوالي • ؛.00 مليار دولار، أما إجمالي قيمة وارداتها الكلية في نفس الفترة فقد

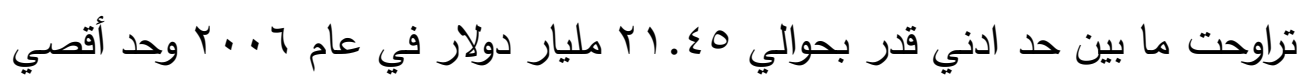

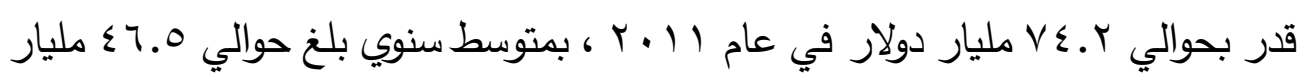
دولار ، وقد حقق الميزان التجاري الكلي اقصي فائض قدر بحوالي ^.9 مليار دولار في

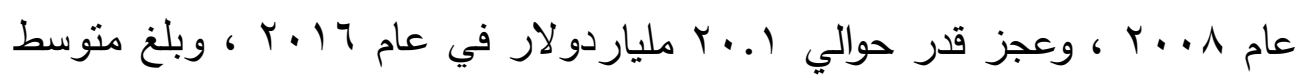
الفائض خلال فترة الدراسة حو الي عـ.^ مليار دولار.

الجدول رقم (1) تطور قيمة الصادرات والواردات الجزائرية خلال الفترة

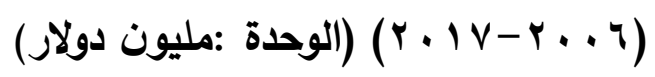

\begin{tabular}{|c|c|c|c|c|c|}
\hline التغطية & التجاريزي & جملة التجارة & الواردات & الصادرات & السنوات \\
\hline 254.5 & 33157 & 76069 & 21456 & 54613 & 2006 \\
\hline 217.7 & 32532 & 87794 & 27631 & 60163 & 2007 \\
\hline 200.8 & 39819 & 118777 & 39479 & 79298 & 2008 \\
\hline 115.01 & 5900 & 84488 & 39294 & 45194 & 2009 \\
\hline 140.9 & 16580 & 97526 & 40473 & 57053 & 2010 \\
\hline 98.9 & -761 & 147733 & 74247 & 73486 & 2011 \\
\hline
\end{tabular}




\begin{tabular}{|c|c|c|c|c|c|}
\hline 142.6 & 21490 & 122242 & 50376 & 71866 & 2012 \\
\hline 117.6 & 9729 & 119697 & 54984 & 64713 & 2013 \\
\hline 100.5 & 326 & 119666 & 59670 & 59996 & 2014 \\
\hline 65.6 & -18083 & 87215 & 52649 & 34566 & 2015 \\
\hline 59.2 & -20126 & 78746 & 49436 & 29310 & 2016 \\
\hline 70.5 & -14411 & 83549 & 48980 & 34569 & 2017 \\
\hline 132.04 & 8846 & 101958.5 & 46556.25 & 55402.25 & المتوسط \\
\hline
\end{tabular}

المصدر : تقارير بنك الجزائر والنشرات الاحصائية.

المطلب الثاني: تطور التركيبة السلعية للصادرات في الجزائر خلال الفترة من

$$
:(r \cdot I V-r \cdot T)
$$

اولأ: تطور التركيبة السلعية للصادرات في الجزائر.

يتضح من الجدول رقم (Y) وشكل رقم (Y) التركيبة السلعية لصادرات الجزائر

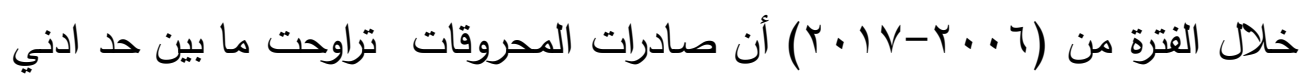
قدر بحوالي PV.

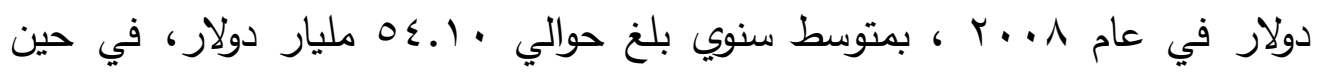
صادرات خارج المحروقات تراوحت ما بين حد ادني قدر بحوالي 7 •. ـ مليار دولار في

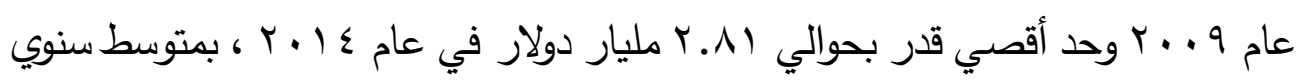

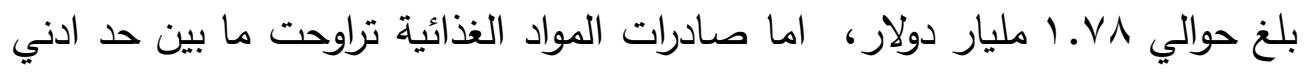

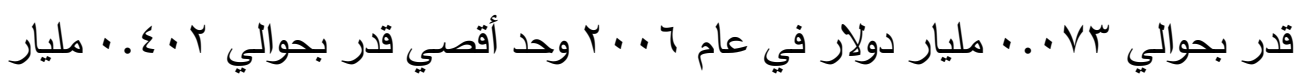




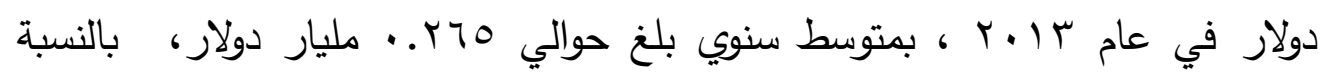
لصادرات المواد الأولية تراوحت ما بين حد ادني قدر بحوالي rV ... مليار دولار في

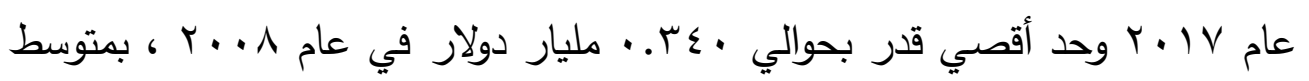
سنوي بلغ حوالي ror or. مليار دولار، اما صادرات التجهيزات الفلاحية لا توجد تقريباً، أما صادرات التجهيزات الصناعية تراوحت ما بين حد ادني قدر بحوالي 9 (... مليار

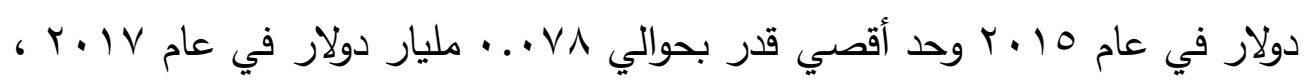
بمتوسط سنوي بلغ حوالي رب+... مليار دولار، في حين صادرات السلع الاستهلاكية

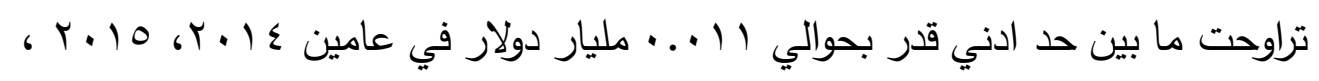
وحد أقصي قدر بحوالي 9 ـ .. . مليار دولار في عام 9 . . ب ، بمتوسط سنوي بلغ حوالي דr... مليار دولار.

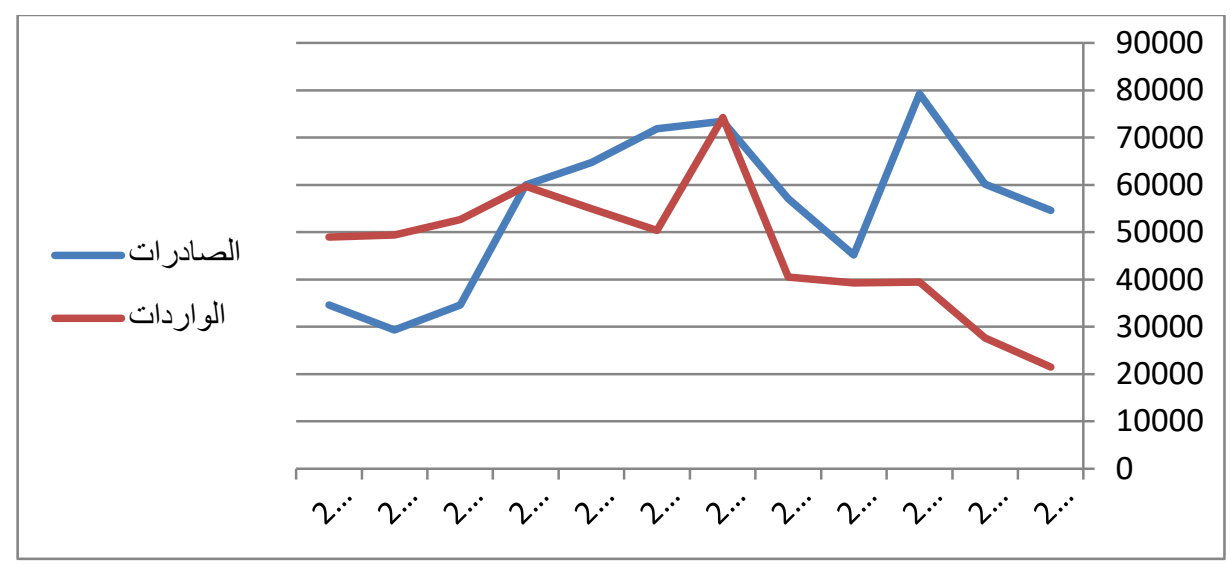

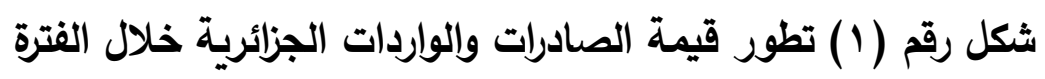

$$
(r \cdot I V-r+. r)
$$




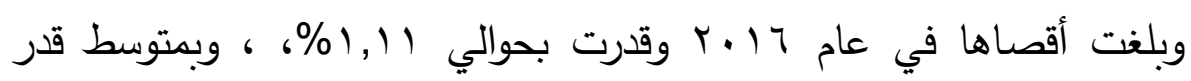
بحواليء0, • \% من إجمالي صادرات الجزائر ـ أما المواد الاولية تحتل المرتبة الثالثة ،

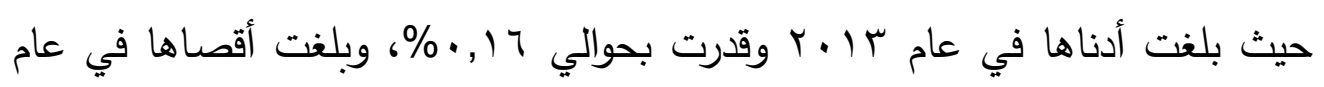

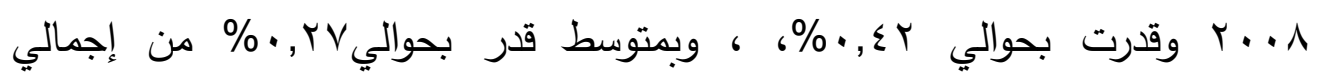
صادرات الجزائر ، بينما احتلت كل من التجهيزات الصناعية والسلع الاستهلاكية المرتبة الرابعة والخامسة من الاهمية النسبية لإجمالي صادرات الجزائر .مما سبق نستتج أن صادرات قطاع المحروقات تأخذ نسبة كبيرة جدا تمثل حوالي و9 من إجمالي الصادرات ، مقارنة بالصادرات خارج المحروقات التي تمثل حوالي ب٪ من إجمالي صادرات الجزائر كما هو موضع بالثكل رقم (r). ثانياً: الاهمية النسبية التركيبة السلعية للصادرات في الجزائر: يتضح من الجدول رقم (ץ) وشكل رقم (ץ) الاهمية النسبية للتركيبة السلعية

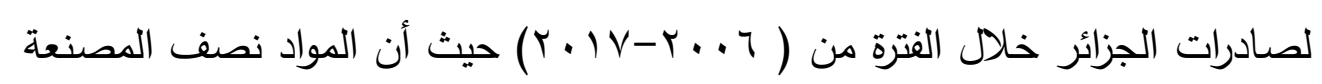
تحتل المرتبة الأولى بالنسبة للمجموعات السلعية الأخرى من حيث أهميتها النسبية، حيث

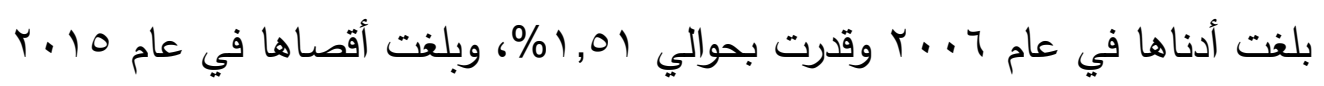

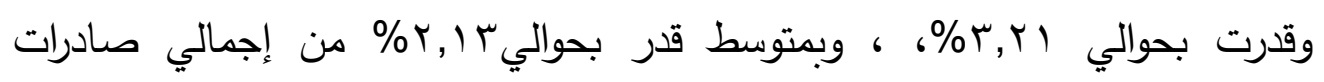
الجزائر. أما المواد الغذائية تحتل المرتبة الثانية وتتمثل أساسا في الحبوب، السكر 
والحليب بالإضافة الي مواد غذائية أخري، حيث بلغت أدناها في عام ج · . r وقدرت

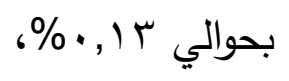

الجدول رقم (Y) تطور التركيبة السلعية للصادرات في الجزائر خلال الفترة من

( مليون دولار) ( $)$

\begin{tabular}{|c|c|c|c|c|c|c|c|c|c|}
\hline الاستهلكية & التجنيزات & التجلاحية & مصنعة & الاولية & الغذائية & المحروقات & المحروقات & الصمالي & السنوات \\
\hline 43 & 44 & 0 & 828 & 195 & 73 & 1183 & 53608 & 54613 & 2006 \\
\hline 43 & 44 & 0 & 988 & 153 & 92 & 1311 & 59506 & 60163 & 2007 \\
\hline 34 & 69 & 0 & 1390 & 340 & 121 & 1954 & 77192 & 79298 & 2008 \\
\hline 49 & 25 & 0 & 692 & 170 & 113 & 1066 & 44411 & 45194 & 2009 \\
\hline 33 & 27 & 0 & 1089 & 165 & 380 & 1619 & 56143 & 57053 & 2010 \\
\hline 16 & 36 & 0 & 1495 & 162 & 357 & 2140 & 71662 & 73486 & 2011 \\
\hline 18 & 30 & 0 & 1519 & 167 & 314 & 2048 & 70571 & 71866 & 2012 \\
\hline 16 & 25 & 0 & 1608 & 108 & 402 & 2161 & 63662 & 64713 & 2013 \\
\hline 11 & 15 & 1 & 1173 & 110 & 323 & 2810 & 58362 & 59996 & 2014 \\
\hline 11 & 19 & 0 & 1111 & 106 & 338 & 1966 & 33081 & 34566 & 2015 \\
\hline 18 & 53 & 0 & 909 & 84 & 326 & 1781 & 27917 & 29310 & 2016 \\
\hline 20 & 78 & 0 & 845 & 73 & 350 & 1367 & 33202 & 34569 & 2017 \\
\hline 26 & 38.75 & 0.08 & 1137.2 & 152.7 & 265.7 & 1783.833 & 54109.75 & 55402.2 & المتوسط \\
\hline
\end{tabular}

المصدر : تقارير بنك الجزائر والنشرات الاحصائية. 


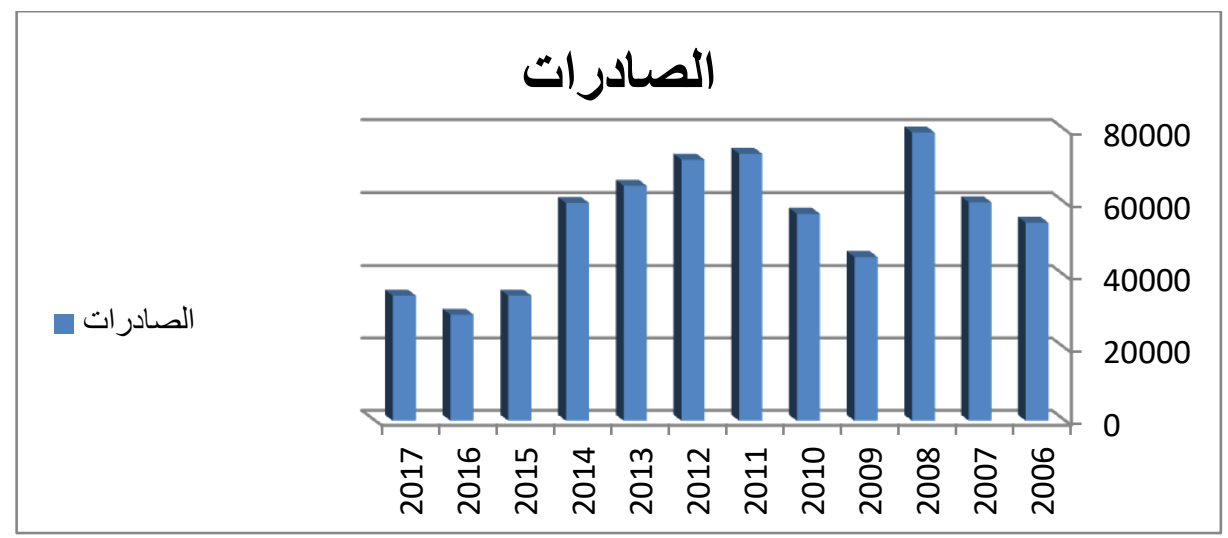

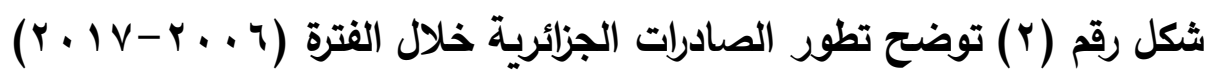

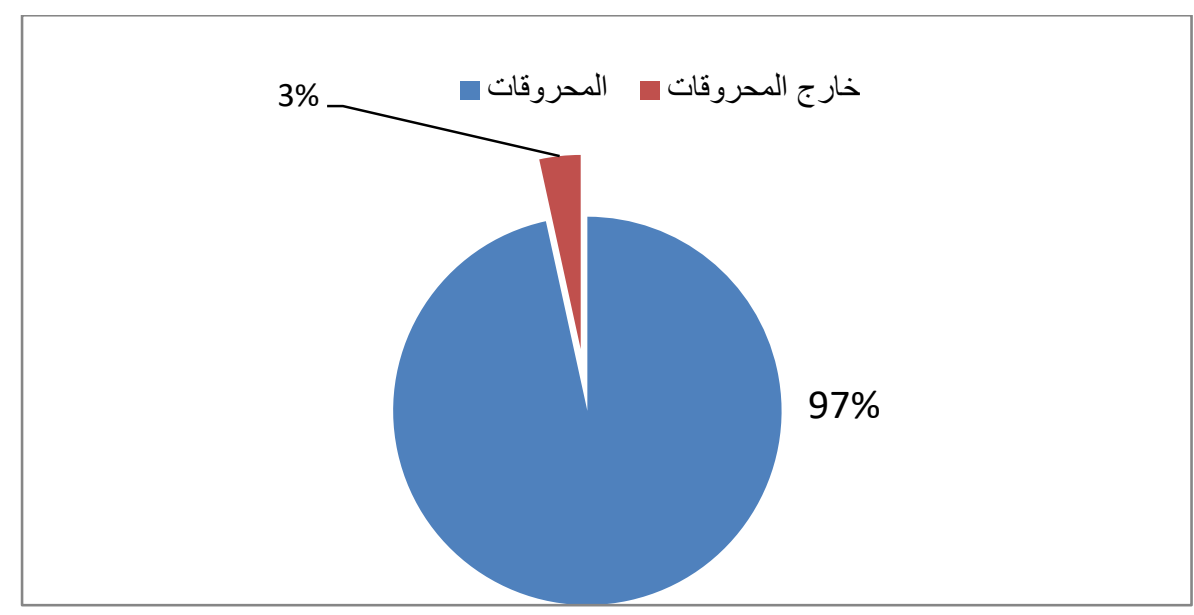

الثكل رقم (ץ) يوضح هيكل التوزيع النسبي لصادرات الجزائر بين المحروقات وخارج المحروقات

الجدول رقم (ץ) الاهمية النسبية التركيبة السلعية للصادرات في الجزائر خلال الفترة

$$
\text { من (r. }
$$




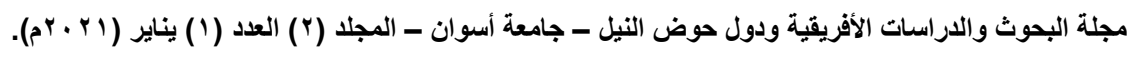

\begin{tabular}{|c|c|c|c|c|c|c|c|c|c|}
\hline السلع & التجهيزات & التجهزات & & المواد & المواد & خارج & الدحروقات & إجمالي & \\
\hline الاستهلاكية & الصناعية & الفلاحية & مصنعة & الاولية & الغذائية & المحروقات & & الصادرات & السنوات \\
\hline 0.078 & 0.08 & 0 & 1.51 & 0.35 & 0.13 & 2.16 & 98.15 & 100 & 2006 \\
\hline 0.071 & 0.07 & 0 & 1.64 & 0.25 & 0.15 & 2.17 & 98.9 & 100 & 2007 \\
\hline 0.04 & 0.087 & 0 & 1.75 & 0.42 & 0.15 & 2.46 & 97.34 & 100 & 2008 \\
\hline 0.11 & 0.05 & 0 & 1.53 & 0.37 & 0.25 & 2.35 & 98.26 & 100 & 2009 \\
\hline 0.05 & 0.047 & 0 & 1.9 & 0.28 & 0.66 & 2.83 & 98.4 & 100 & 2010 \\
\hline 0.02 & 0.048 & 0 & 2.03 & 0.22 & 0.48 & 2.91 & 97.51 & 100 & 2011 \\
\hline 0.025 & 0.041 & 0 & 2.11 & 0.23 & 0.43 & 2.84 & 98.19 & 100 & 2012 \\
\hline 0.024 & 0.038 & 0 & 2.48 & 0.16 & 0.62 & 3.33 & 98.37 & 100 & 2013 \\
\hline 0.018 & 0.025 & 0 & 1.95 & 0.18 & 0.53 & 4.68 & 97.27 & 100 & 2014 \\
\hline 0.031 & 0.054 & 0 & 3.21 & 0.31 & 0.97 & 5.68 & 95.7 & 100 & 2015 \\
\hline 0.061 & 0.18 & 0 & 3.1 & 0.28 & 1.11 & 6.07 & 95.24 & 100 & 2016 \\
\hline 0.057 & 0.22 & 0 & 2.44 & 0.21 & 1.01 & 3.95 & 96.04 & 100 & 2017 \\
\hline 0.05 & 0.078 & 0 & 2.135 & 0.27 & 0.54 & 3.45 & 97.45 & 100 & المتوسط \\
\hline
\end{tabular}

المصدر : حسبت من بيانات جدول رقم (r)

\section{المطلب الثالث: تطور التركيبة السلعية للواردات في الجزائر خلال الفترة من$$
: Y \cdot I V-r \cdot . q
$$

إن الحديث عن الواردات يعني أن حجم الإنتاج المحلي لم يستطع تلبية

احتياجات المستهلك، وقد يعود عدم القدرة هذا إلى قلة حجم الانتاج، لسعره أو لنوعيته، 
أو لأن الوضع الاقتصادي الحالي المتميز بتسهيل الواردات جعل هذه الأخيرة محل تفضيل على المنتوج المحلي حتى ولو كان هذا الأخير في درجة من الجودة.

اولأ: تطور التركيبة السلعية للواردات في الجزائر:

يتضح من الجدول رقم (£) وشكل رقم (£) التركيبة السلعية لواردات الجزائر

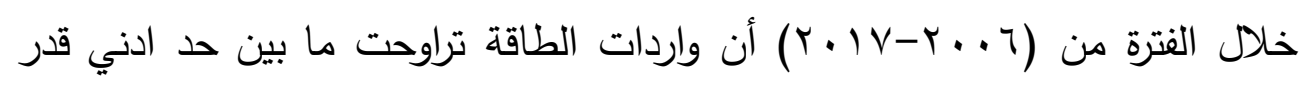

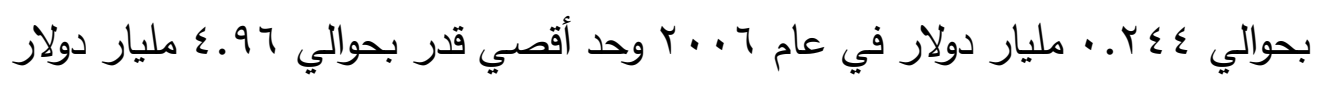

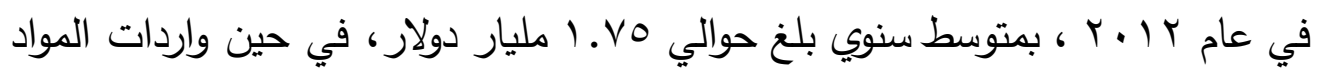
الغذائية تراوحت ما بين حد ادني قدر بحوالي ^.r مليار دولار في عام ؟ .. ب وحد

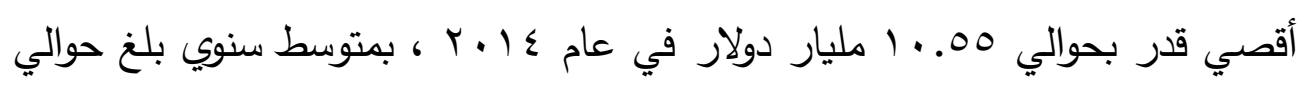
ع.7. Vليار دولار ، اما واردات المواد الاولية تراوحت ما بين حد ادني قدر بحوالي

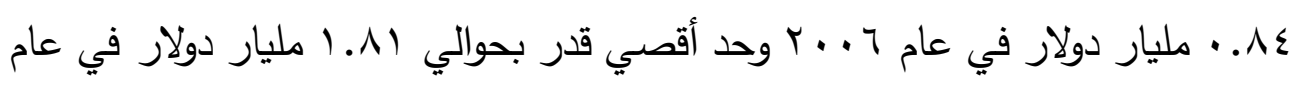

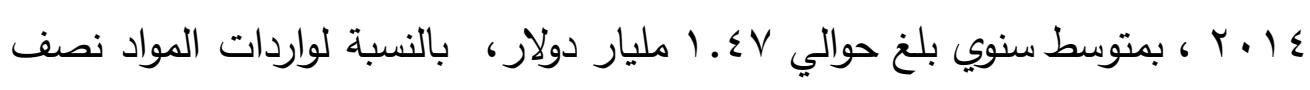

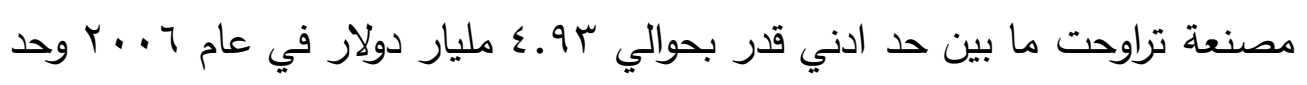

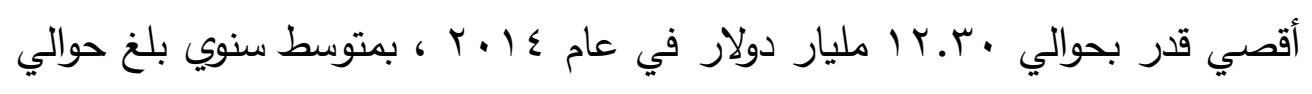

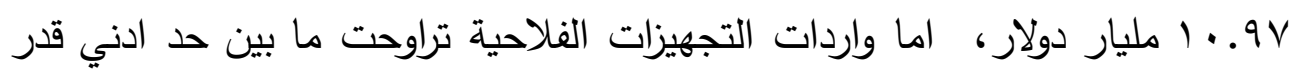

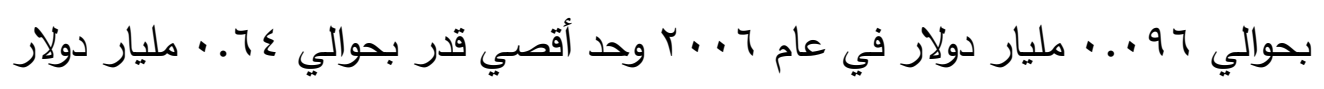


في عام 0 • ب ، بمتوسط سنوي بلغ حوالي هب. · مليار دولار، أما واردات التجهيزات

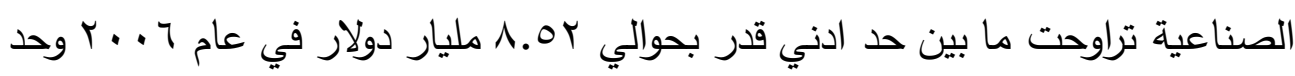

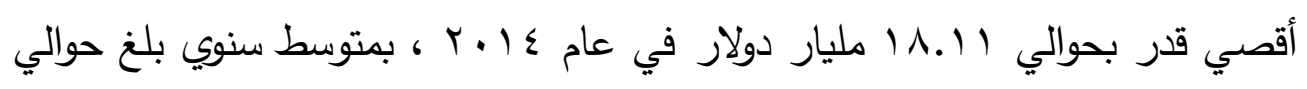
بس.؛ ( مليار دولار، في حين واردات السلع الاستهلاكية تراوحت ما بين حد ادني قدر

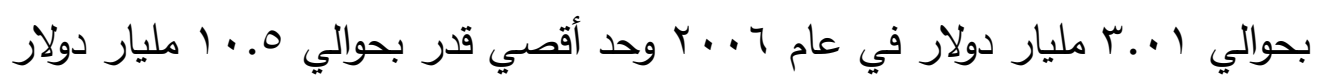

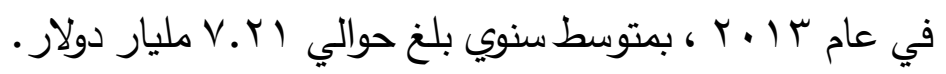
ثانياً: الاهمية النسبية التركيبة السلعية للواردات في الجزائر: يتضح من الجدول رقم (0) وشكل رقم (0) الاهمية النسبية للتركيبة السلعية

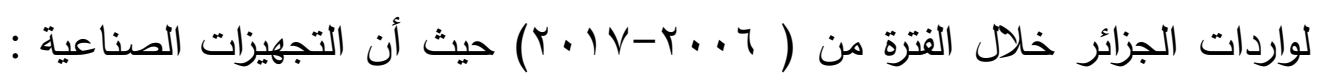
تحتل المرتبة الأولى بالنسبة للجموعات السلعية الأخرى من حيث أهميتها حيث تحظى سلع التجهيز الصناعية كل سنة بنسب معتبرة من اجمالي الواردات، لكونها ضرورية لمواصلة عملية الاستراتيجية التنموية التي اتخذتها الدولة للنهوض بالاقتصاد، خاصة لخدمة التصنيع من أجل التصدير لتشجيع صادارتها، والمتمثلة أساسا في الآلات

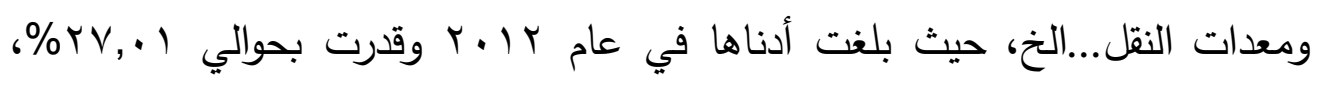

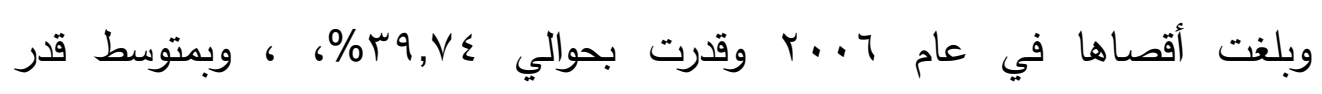

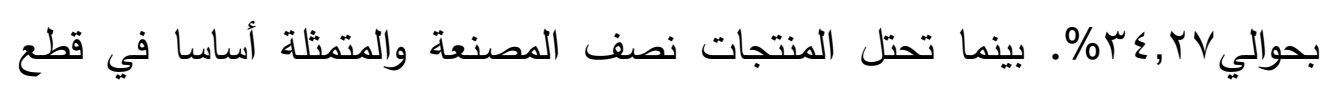
الغيار ومنتجات أخري المرتبة الثانية في قائمة المواد المستوردة بعد سلع التجهيزات 
الصناعية، فهي من بين المنتجات اللازمة لمواصلة تشغيل عجلات الانتاج في مختلف قطاعات الاقتصاد الوطني، حيث بلغت أدناها في عام r. r وقدرت بحوالي

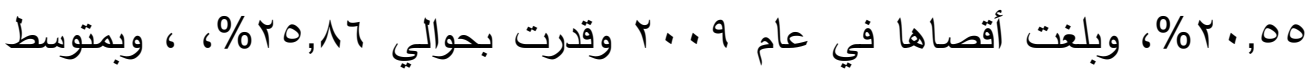
قدر بحوالي اr,r\%\%. أما المواد الغذائية تحتل المرتبة الثالثة وتتمثل أساسا في الحبوب، السكر والحليب بالإضافة الي مواد غذائية أخري، حيث بلغت أدناها في عام

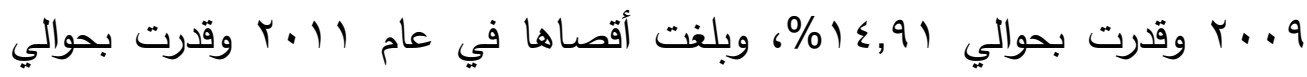

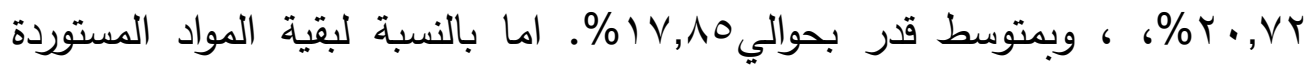
كالسلع الاستهلاكية والطاقة والمواد الاولية فتحتل المرتبة الرابعة والخامسة والسادس من

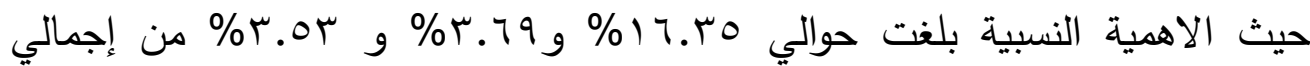
الواردات علي التوالي، بينما احتلت التجهيزات الفلاحية المرتبة الاخيرة من حيث الاهمية النسبية وبلغت حوالي V9. • \% من إجمالي الواردات. الجدول رقم (ع ) تطور التركيبة السلعية للواردات في الجزائر خلال الفترة من

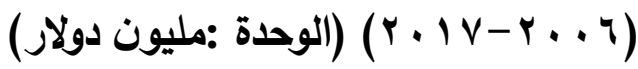

\begin{tabular}{|c|c|c|c|c|c|c|c|c|}
\hline الاستهلاكية & التجنيزاتة & الفلاحهزات & مصنعة & الاولية & الغذائية & الطاقة & الجمالي & السنو ات \\
\hline 3011 & 8528 & 96 & 4934 & 843 & 3800 & 244 & 21456 & 2006 \\
\hline 3752 & 10026 & 146 & 7105 & 1325 & 4954 & 324 & 27631 & 2007 \\
\hline 5036 & 15434 & 86 & 9154 & 1378 & 7796 & 595 & 39479 & 2008 \\
\hline 6145 & 15140 & 234 & 10165 & 1201 & 5863 & 549 & 39297 & 2009 \\
\hline
\end{tabular}




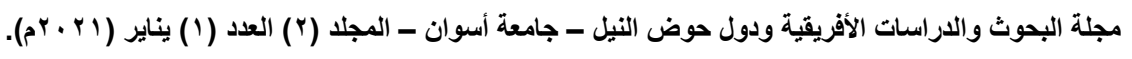

\begin{tabular}{|c|c|c|c|c|c|c|c|c|}
\hline 5987 & 15573 & 330 & 9944 & 1404 & 6027 & 945 & 40212 & 2010 \\
\hline 7944 & 15951 & 229 & 10431 & 1776 & 9805 & 1164 & 47300 & 2011 \\
\hline 9997 & 13604 & 329 & 10629 & 1839 & 9023 & 4955 & 50376 & 2012 \\
\hline 10539 & 15233 & 477 & 10642 & 1732 & 9013 & 4139 & 51775 & 2013 \\
\hline 9894 & 18115 & 629 & 12301 & 1812 & 10550 & 2720 & 56021 & 2014 \\
\hline 8243 & 16369 & 638 & 11482 & 1489 & 8946 & 2247 & 49414 & 2015 \\
\hline 7904 & 14709 & 479 & 10972 & 1490 & 7855 & 1234 & 44643 & 2016 \\
\hline 8129 & 13368 & 585 & 10483 & 1456 & 8069 & 1899 & 43989 & 2017 \\
\hline 7215.1 & 14337.5 & 354.8 & 9853.5 & 1478.7 & 7641.7 & 1751.25 & 42632.7 & (المتوس \\
\hline
\end{tabular}

المصدر : تقارير بنك الجزائر والنشرات الاحصائية.

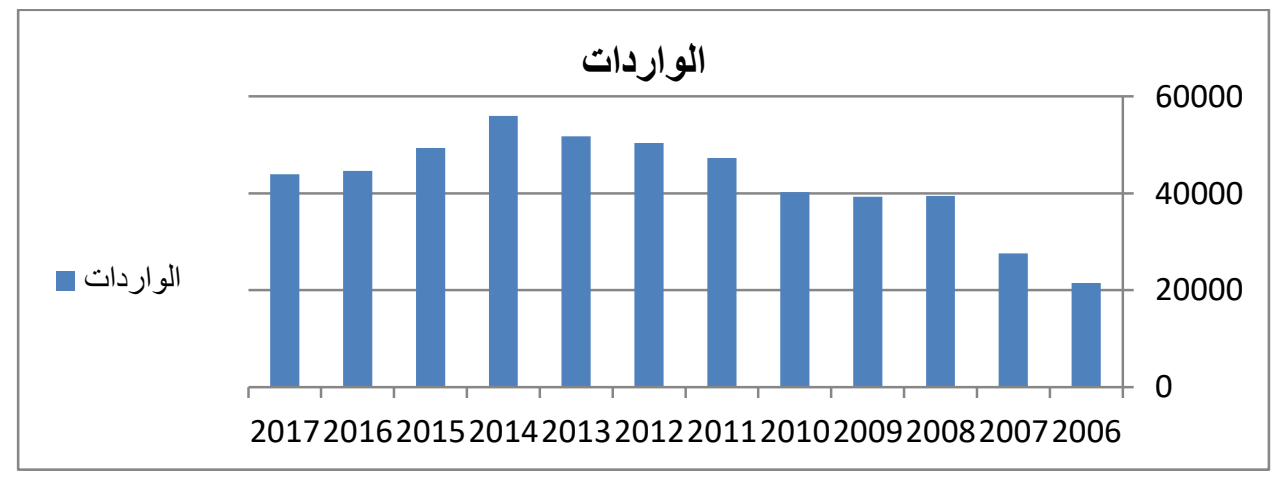

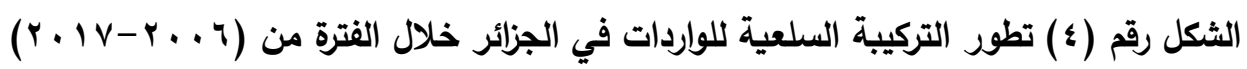

الجدول رقم (0) الاهمية النسبية التركية السلعية للواردات في الجزائر خلال

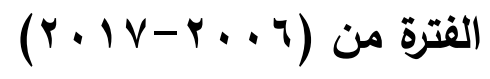

\begin{tabular}{|c|c|c|c|c|c|c|c|c|}
\hline السلع الاستهلاكية & التجناعيزات & الفلاحية & مصن & الالولية & الغذائية & الطاقة & إلجمالي & السنوات \\
\hline 14.03 & 39.74 & 0.44 & 22.99 & 3.928 & 17.71 & 1.13 & 100 & 2006 \\
\hline 13.57 & 36.28 & 0.52 & 25.71 & 4.79 & 17.92 & 1.172 & 100 & 2007 \\
\hline 12.75 & 39.09 & 0.21 & 23.18 & 3.490 & 19.74 & 1.507 & 100 & 2008 \\
\hline 15.63 & 38.52 & 0.59 & 25.86 & 3.056 & 14.91 & 1.39 & 100 & 2009 \\
\hline
\end{tabular}




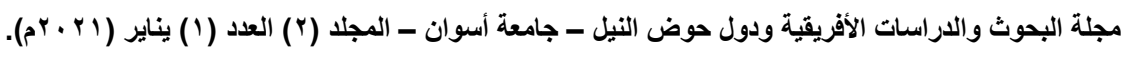

\begin{tabular}{|c|c|c|c|c|c|c|c|c|}
\hline 14.88 & 38.72 & 0.82 & 24.76 & 3.491 & 14.98 & 2.35 & 100 & 2010 \\
\hline 16.79 & 33.72 & 0.48 & 22.05 & 3.75 & 20.72 & 2.46 & 100 & 2011 \\
\hline 19.84 & 27.01 & 0.65 & 21.09 & 3.65 & 17.91 & 9.83 & 100 & 2012 \\
\hline 20.35 & 29.42 & 0.92 & 20.55 & 3.34 & 17.40 & 7.99 & 100 & 2013 \\
\hline 17.66 & 32.33 & 1.12 & 21.95 & 3.23 & 18.83 & 4.85 & 100 & 2014 \\
\hline 16.68 & 33.12 & 1.29 & 23.23 & 3.01 & 18.10 & 4.54 & 100 & 2015 \\
\hline 17.70 & 32.94 & 1.07 & 24.57 & 3.33 & 17.59 & 2.76 & 100 & 2016 \\
\hline 18.47 & 30.38 & 1.32 & 23.83 & 3.30 & 18.34 & 4.31 & 100 & 2017 \\
\hline 16.53 & 34.27 & 0.79 & 23.31 & 3.53 & 17.85 & 3.69 & 100 & المتوسط \\
\hline
\end{tabular}

المصدر : حسبت من بيانات جدول رقم (r)

المبث الثاني: التوزيع الجغرافي لصادرات و واردات الجزائر حسب المناطق

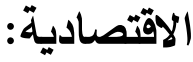

المطلب الاول: التوزيع الجغرافي للواردات الجزائريـة:

تشير بيانات الجدول رقم (7) أن دول الاتحاد الاوربي تأتي في المرتبة الاولي

من حيث الواردات الجزائرية، حيث أخذت قيمة الواردات من الاتحاد الاوربي، حيث

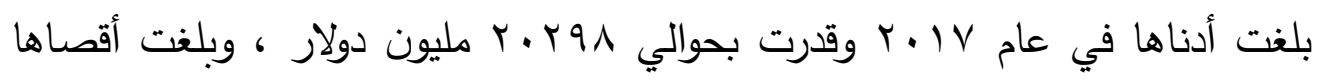

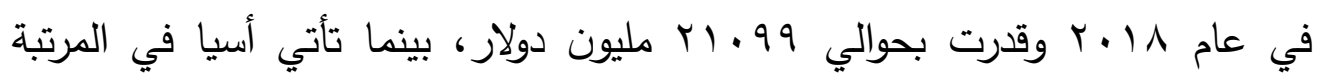

الثانية بعد الاتحاد الأوربي من ناحية تموين الاقتصاد الجزائري، حيث بلغت أدناها في

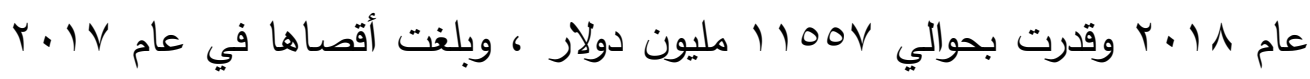

وقدرت بحوالي ج4س ا مليون دولار، اما دول منظمة التعاون والتتمية الاقتصادية 


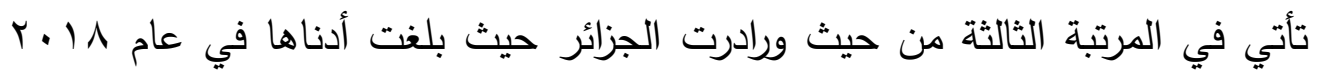

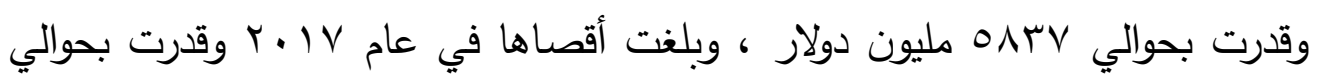
rrqه مليون دولار، أما بالنسبة لبقية المناطق الاقتصادية كانت قيم واردات الجزائر تتذبذب بين الارتفاع والانخفاض حيث بلغت أدناها من الدول الافريقية بحوالي 177 مليون دولار ، واقصاها من دول امريكا الجنوبية حيث بلغت حوالي 7 ــ مليون دولار عام 11.

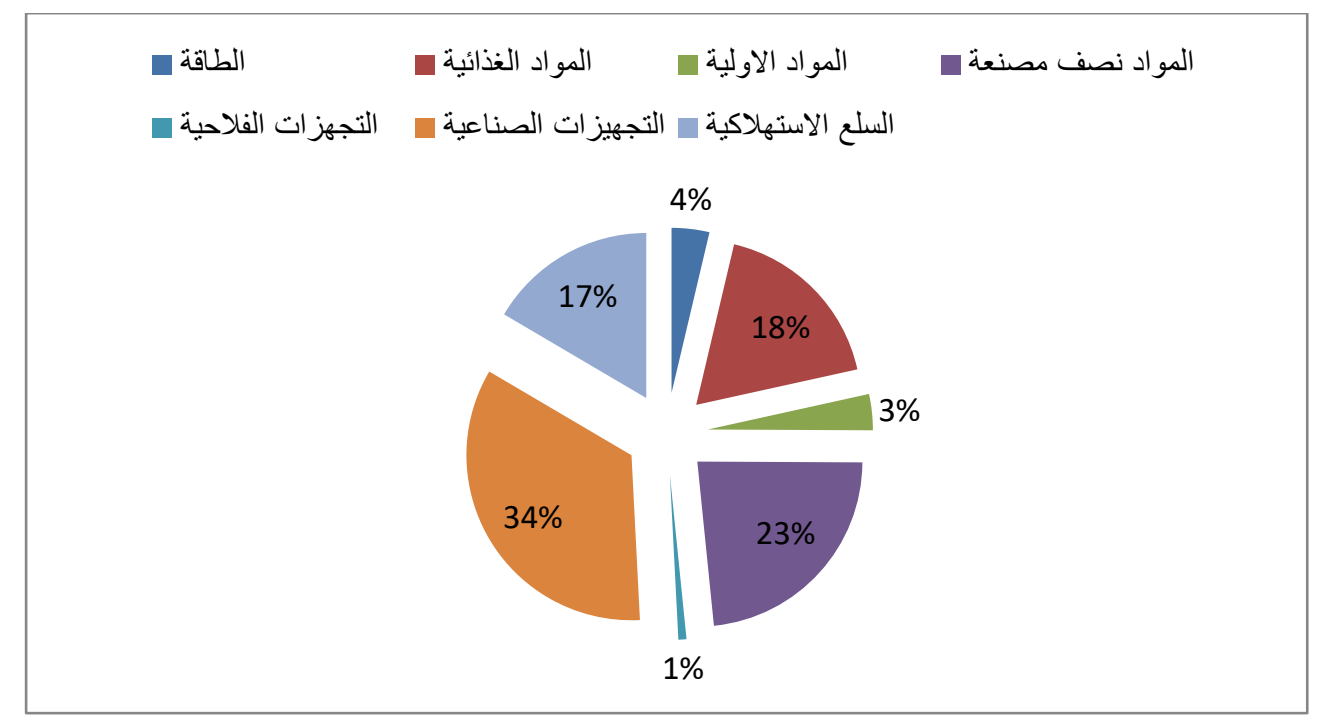

شكل رقم (ه) الاهمية النسبية التركيبة السلعية للواردات في الجزائر

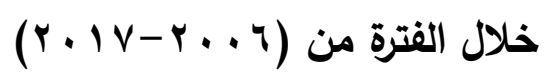

المطلب الثاني التوزيع الجغرافي للصادرات الجزائرية: تتنوع الصادرات الجزائرية علي شريحة واسعة من المتعاملين، تشير بيانات الجدول رقم (؟) والذي يبين مجموعات الدولية التي تمثل أسواق للجزائر في الخارج، 
حيث تحتل دول الاتحاد الاوربي المرتبة الاولي من حيث الصادرات الجزائرية، حيث أخذت قيمة الصادرات من الاتحاد الاوربي، حد أدني في عام V ا. r وقدرت بحوالي

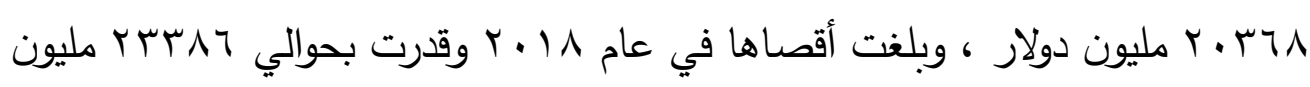
دولار ، اما دول منظمة التعاون والتتمية الاقتصادية تأتي في المرتبة الثانية من حيث

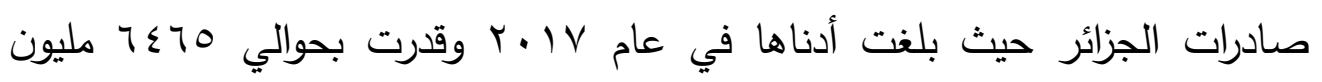
دولار ، وبلغت أقصاها في عام 11 ـ ب وقدرت بحوالي .790 مليون دولار، بينما تأتي أسيا في المرتبة الثالثة من حيث صادرات الجزائر ، حيث بلغت أدناها في عام Y. V r. T

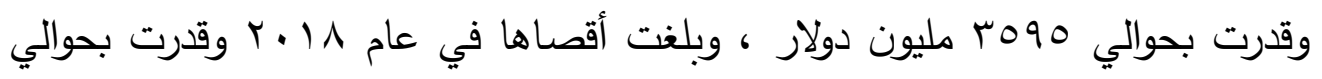
10rol مليون دولار، ، أما بالنسبة لبقية المناطق الاقتصادية كانت قيم صادرات الجزائر تتذبذب بين الارتفاع والانخفاض حيث بلغت أدناها من الدول الافريقية بحوالي r Tا مليون دولار ، واقصاها من دول امريكا الجنوبية حيث بلغت حوالي . بTr مليون دولار عام 11.r.

المطلب الثالث: أهم شركاء الجزائر للصادرات خلال عام 11 ـ ؟ ؟ يظهر لنا الجدول رقم (V) والثكل رقم (V) أهم الدول المتعاملة مع الجزائر في مجال الصادرات، ويوضح أهم الدول التي تمثل أسواق للجزائر في الخارج ، نلاحظ أن ايطاليا تحتل صدارة الدتعاملين مع الجزائر في إطار الصادرات لعام 1 بـr حيث

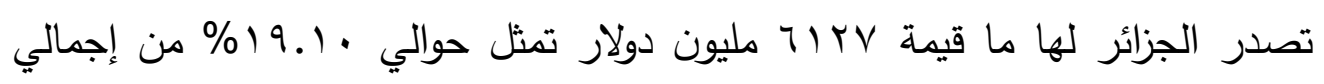


الصادرات، ويليها في الترتيب كل من اسبانيا وفرنسا والولايات المتحدة الامريكية

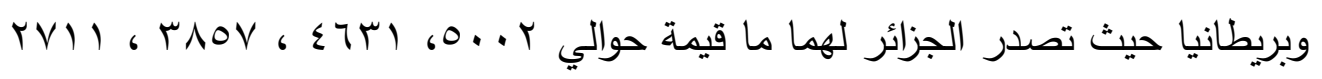

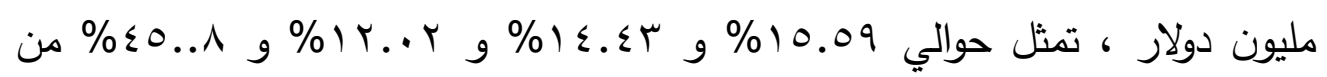
إجمالي الصادرات علي الترتيب،

الجدول رقم (؟) توضح التوزيع الجغرافي للواردات والصادرات حسب المناطق الاقتصادية

\begin{tabular}{|c|c|c|c|c|}
\hline \multicolumn{2}{|c|}{ الصادرات (مليون دولار ) } & \multicolumn{2}{|c|}{ الواردات (مليون دولار) } & \multirow[t]{2}{*}{ الدناطق الاقتصادية } \\
\hline 2018 & 2017 & 2018 & 2017 & \\
\hline 23386 & 20368 & 21099 & 20298 & دول الاتحاد الاوربي \\
\hline 6950 & 6465 & 5837 & 5933 & دولتمل منظمة التعاون \\
\hline 40 & 40 & 1542 & 1910 & الدول الاوربية \\
\hline 2660 & 2530 & 3546 & 3209 & دول امريكا الجنوبية \\
\hline 5351 & 3595 & 11557 & 12369 & اسيا \\
\hline 712 & 799 & 1904 & 1542 & الدول العربية \\
\hline 1669 & 1273 & 546 & 592 & الدول المغاربية \\
\hline 132 & 103 & 166 & 186 & الدول الافريقية \\
\hline
\end{tabular}




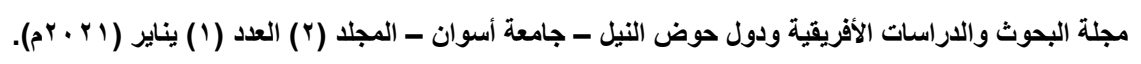

\begin{tabular}{|l|l|l|l|l|}
\hline 40900 & 35173 & 46197 & 46039 & المجموع \\
\hline
\end{tabular}

المصدر : المركز الوطني للاعلام الالي والاحصاء التابع للجمارك.

اما المراتب الخمس الاخيرة جاءت كل من تركيا و هولندا و البرازيل و الهند

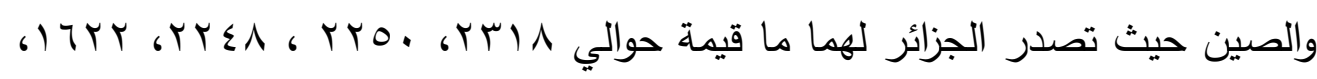

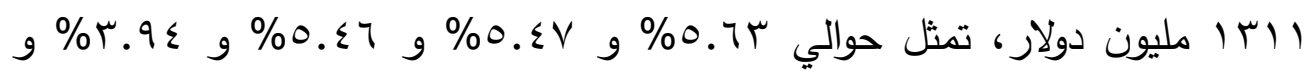
1 ا.ب\% من إجمالي الصادرات علي الترتيب.

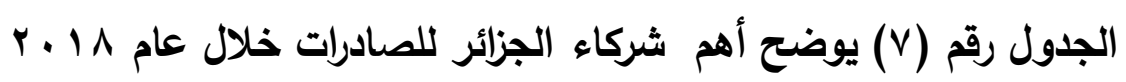

\begin{tabular}{|c|c|c|c|}
\hline$\%$ & القيمة الق & 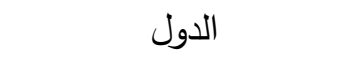 & الرقم \\
\hline 19.10 & 6127 & ايطاليا & 1 \\
\hline 15.59 & 5002 & اسبانيا & 2 \\
\hline 14.43 & 4631 & فرنسا & 3 \\
\hline 12.02 & 3857 & الولايات المتحدة الامريكية & 4 \\
\hline 8.45 & 2711 & بريطانيا & 5 \\
\hline 7.22 & 2318 & تركيا & 6 \\
\hline 7.01 & 2250 & هولندا & 7 \\
\hline 7.01 & 2248 & البرازيل & 8 \\
\hline 5.05 & 1622 & الهند & 9 \\
\hline 4.08 & 1311 & الصين الصين & 10 \\
\hline
\end{tabular}




\begin{tabular}{|l|l|l|c|}
\hline 100 & 32077 & & المجموك \\
\hline
\end{tabular}

المصدر : المركز الوطني للمعلومات الاحصائية للجمارك.

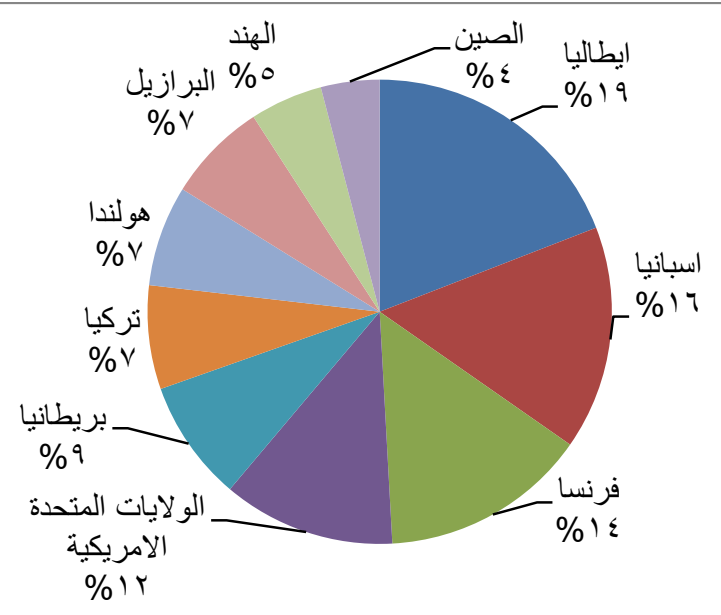

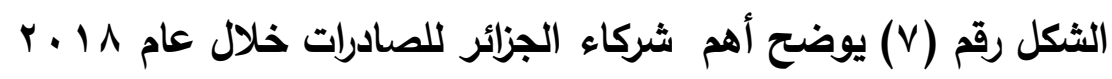

المطلب الرابع: أهم شركاء الجزائر للواردات خلال عام 1 ـ ـ ؟: يظهر لنا الجدول رقم (^) والثكل رقم (^) أهم الدول المتعاملة مع الجزائر في مجال الاستيراد، إذ يمكن من خلال معرفة مدي ارتباط الجزائر بالأسواق الخارجية ومعرفة ددي الاعتماد علي دولة واحدة أو تكتل اقتصادي واحد في جلب المنتجات التي بحاجة اليها ، نلاحظ أن الصين تحتل صدارة المتعاملين مع الجزائر في إطار

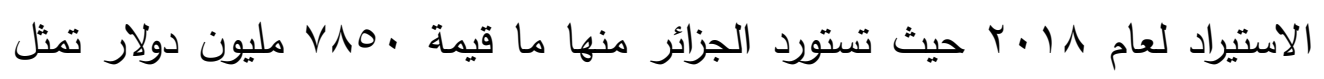
حوالي ج ×.0\% من إجمالي الواردات، ويليها في الترتيب كل من فرنسا وايطاليا

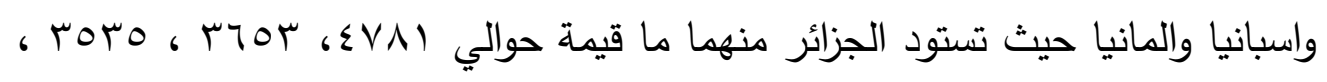

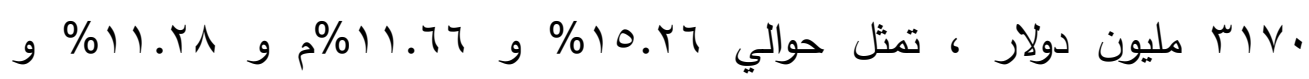


ץ... (1\% من إجمالي الواردات علي الترتيب، اما المراتب الخمس الاخيرة جاءت كل من تركيا و الارجنتين و الولايات المتحدة الامريكية و الهند و كوريا حيث تستود

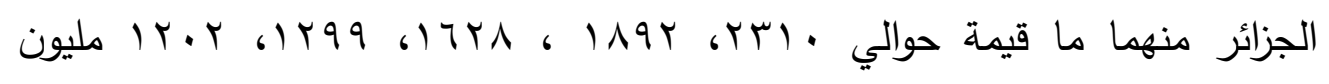

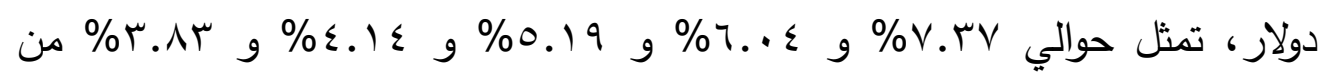
إجمالي الواردات علي الترتيب.

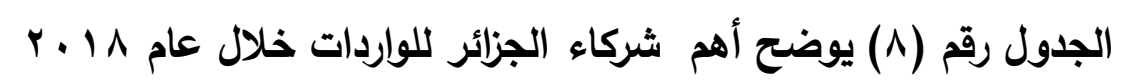

\begin{tabular}{|c|c|c|c|}
\hline$\%$ & القيمة & الدول & الرقم \\
\hline 25.06 & 7850 & الصين & 1 \\
\hline 15.26 & 4781 & فرنسا & 2 \\
\hline 11.66 & 3653 & ايطاليا & 3 \\
\hline 11.28 & 3535 & اسبانيا & 4 \\
\hline 10.12 & 3170 & المانيا & 5 \\
\hline 7.37 & 2310 & تركيا & 6 \\
\hline 6.04 & 1892 & الارجنتين & 7 \\
\hline 5.19 & 1628 & الولايات الدنحدة الامريكية & 8 \\
\hline 4.14 & 1299 & الهند & 9 \\
\hline 3.83 & 1202 & كوريا & 10 \\
\hline 100 & 31320 & & الهجموع \\
\hline
\end{tabular}

المصدر : المركز الوطني للمعلومات الاحصائية للجمارك. 
مجلة البحوث والدراسات الأفريقية ودول حوض النيل - جامعة أسوان - المجلد (r) العدد ( ) يناير ( r + rم).

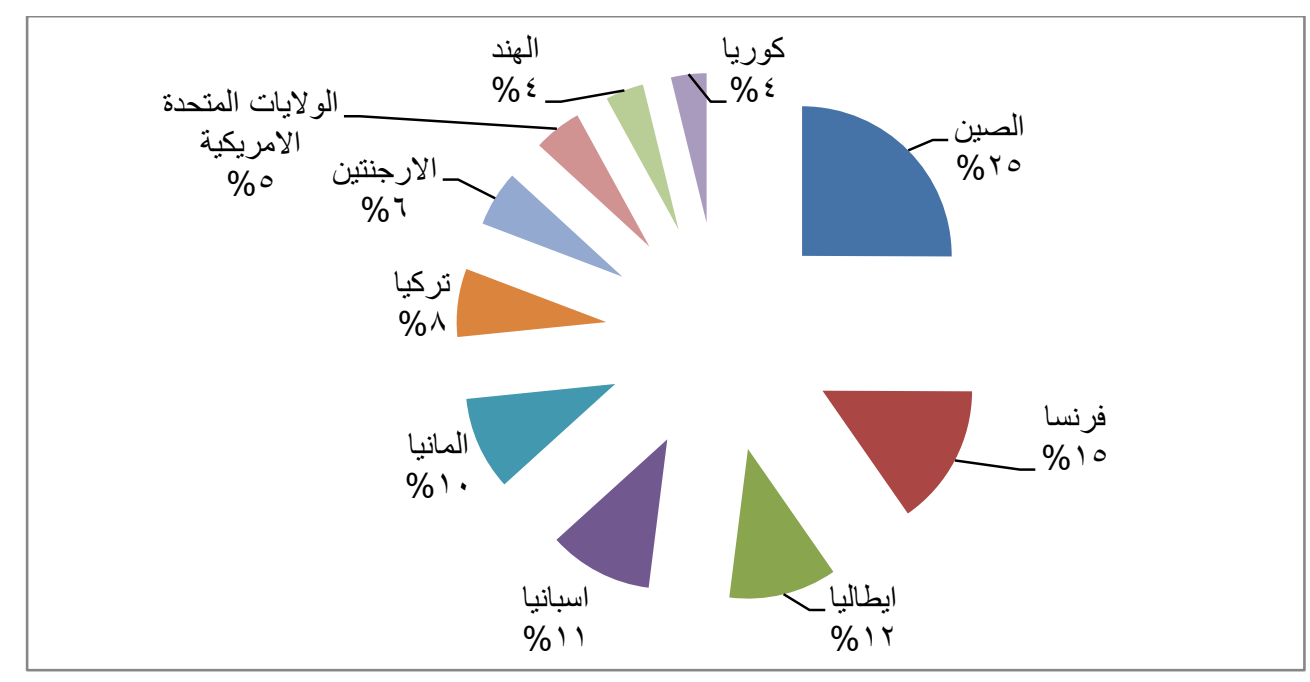

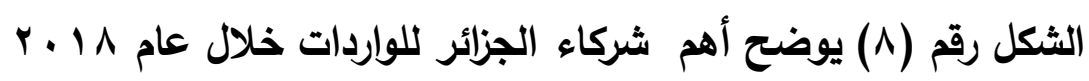




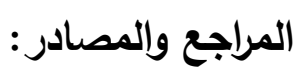

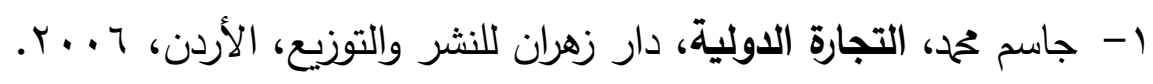

ץ- السيد محم أحمد السريني، التجارة الخارجية، الدار الجامعية، الاسكندارية، 9 . ـ. r-زبير طيوح، أثر التجارة الخارجية علي الميزان التجاري دراسة حالة الجزائر

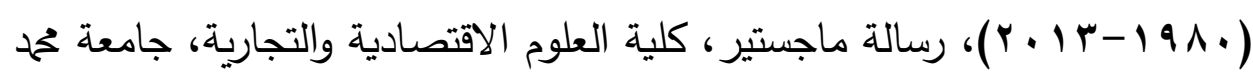

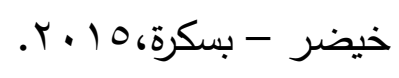

؟- زيرمي نعيمة، التجارة الخارجية الجزائرية في منطقة التجارة الحرة العربية الكبري،

المجلة الجزائرية للاقتصاد والادارة، جامعة بشار ، 17 ب r

http://www.douane.gov.dz

$$
\text { 0- - العامة للجمارك الجزائرية على الموقع }
$$

$$
\text { الإلكتروني }
$$

